\title{
Spirotetramat is toxic to leafrollers on kiwifruit
}

\author{
K.A. Stannard ${ }^{1}$ and M.G. Hill ${ }^{2}$ \\ ${ }^{1}$ Plant E Food Research, 412 No1 Rd, RD2 Te Puke, New Zealand \\ ${ }^{2}$ Plant \& Food Research, Private Bag 4704, Christchurch, New Zealand \\ Corresponding author: garry.hill@plantandfood.co.nz
}

Spirotetramat is an ambimobile selective insecticide used widely in the horticulture industry for controlling a broad range of sucking pests in perennial and annual crops. It has become the standard chemical for the control of armoured scale insects in kiwifruit. During an experiment to test the effects of spirotetramat (formulated as Movento ${ }^{\mathrm{TM}}$ 100SC) on phytohormone levels it unexpectedly caused high levels of mortality to neonate brown-headed leafroller (Ctenopseustis obliquana) larvae ( $96 \%$ of treated plants with dead larvae compared with $9 \%$ in unsprayed controls) exposed one day after spraying to runoff on small potted $A$. deliciosa 'Hayward' plants. Repeating the experiment with different plants and third instar larvae resulted in $98 \%$ treatment mortality compared with $29 \%$ on untreated controls. A third experiment using neonate larvae applied to the same plants as the second experiment 10 days after the spray application, resulted in $76 \%$ treatment mortality compared with $20 \%$ on control plants. Spirotetramat can only be used pre-flowering and leafroller control sprays are applied principally during the first 6 weeks after fruit set. However, the potential contribution of spirotetramat to leafroller control on kiwifruit should be investigated further.

\section{Exploring potential non-target impacts of spinetoram against beneficial natural enemies of Eucalyptus forests}

\author{
A.R. Pugh, M.W. Davis, M.C. Watson and T.M. Withers \\ Scion, Private Bag 3020, Rotorua 3046, New Zealand \\ Corresponding author: Andrew.Pugh@scionresearch.com
}

Forest Stewardship Council-certified Eucalyptus nitens plantations are seeking an alternative insecticide that is more compatible with biological control agents than the current broad-spectrum insecticide used. Various methods were trialled for evaluating spinetoram on beneficials, including Enoggera nassaui, an egg parasitioid of Paropsis charybdis, Cleobora mellyi, a predatory ladybird, and Cotesia urabae, a larval parasitoid of Uraba lugens. While eggs could be directly sprayed, very mobile larvae and adults instead had to be transferred onto recently-sprayed foliage. For ladybirds, the assay environment was supplemented with an artificial diet to avoid mortality from starvation. These practical challenges limit the ability to confidently draw conclusions about impacts in the field. Despite the limitations, it can be reported that $C$. mellyi suffered no mortality from exposure to spinetoram, and females continued to lay viable eggs. In contrast, the endoparasitoid C. urabae, did not survive spinetoram treatment due to the rapid mortality of its host. Both $P$. charybdis eggs and their parasitoids developing within them appeared unharmed by spinetoram. These mixed results suggest spinetoram does have potential as a possible replacement for alpha-cypermethrin, although further investigation of efficacy in the field is needed. 